\title{
Vision inspection system for the identification and classification of defects in MIG welding joints
}

\author{
G. Senthil Kumar • U. Natarajan • S. S. Ananthan
}

Received: 2 October 2010 / Accepted: 14 November 2011 /Published online: 9 December 2011

(C) The Author(s) 2011. This article is published with open access at Springerlink.com

\begin{abstract}
The variety of vision inspection systems for welding defects in the present manufacturing scenario is needed for overcoming certain limitations such as the problem of inaccuracy in the images, non-uniformed illumination, noise and deficient contrast, and confusion in defects if they occur in the same spot at the surface and subsurface. Hence, it is imperative to design a new vision inspection system which will enable to overcome the aforementioned problems in welding. A sophisticated new vision inspection system using machine vision has been developed for this study to identify and classify the surface defects of butt joint as per standard EN25817 in metal inert gas (MIG) welding. In this proposed vision system, images of welding surfaces are captured through a CCD camera. Four frames of sequence of images are obtained using four zones of LEDs using the front light illumination system in this method. From these images, the regions of interest are segmented and the average gray levels of the characteristic features of these images are calculated. The same process can be extended further to four zones (four quadrants) of four types of welded joints. Finally, welded joints can be classified into one of the four predefined ones based on the back-propagation neural network. The proposed system
\end{abstract}

G. Senthil Kumar ( $₫)$

Department of Mechanical Engineering,

Velammal College of Engineering \& Technology,

Madurai 625009 Tamil Nadu, India

e-mail: senthilgandhi@ymail.com

U. Natarajan

Department of Mechanical Engineering,

A. C. College of Engineering \& Technology,

Karaikudi 630004 Tamil Nadu, India

S. S. Ananthan

Welding Research Institute, Bharat Heavy Electricals Ltd.,

Tiruchirapalli 620014, India demonstrates an overall accuracy of $95 \%$ from the 80 real samples tested.

Keywords Machine vision - Weld classification - Industrial inspection · Back-propagation neural network (BPN)

\section{Introduction}

Over the last four decades, the worldwide manufacturing markets have been facing heavy competition to produce cost-effective higher quality products. This has led to great advances in the technology required for automating production processes, but problems in inspection and quality control are yet to be fully resolved. Due to these gaps in the industry, there arises the necessity for active research in inspection and quality control. Inspection of the quality of weld is performed using various non-destructive tests. Though humans (experts) can do better than machines in many ways in the content of visual inspection and quality control, they get tired quickly and the process becomes slow. Human inspection of weld defects is a hard and difficult task when great numbers of welds are to be counted and inspected. Many inspection tasks are considered time-consuming and boring for humans to perform. It has been reported that human visual inspection is estimated to account for $10 \%$ or more of the total labor costs of manufactured products [1]. Moreover, human experts are difficult to find or to maintain their training, and their skills may take time to develop. Machine vision may effectively replace human inspection in such demanding cases. Nondestructive testing (NDT) is a branch of engineering concerned with the methods of detecting defects in objects without altering the object in any way. The reliable detection of weld defects is one of the most important tasks in nondestructive testing. Improvements in these methods are 
necessary because the human factor still has great influences on the evaluation. Welding is a major joining process used to fabricate many engineered artifacts and structures such as cars, ships, space shuttles, offshore drilling plate forms, and pipelines [2]. Shafeek et al. [1] introduced a novel automated vision system to detect and assess the welding defects of gas pipelines from radiographic films. This vision system, used to capture images for the radiographic films, can apply various image processing and computer vision algorithms to detect welding defects and calculate necessary information such as length, width, area, and perimeter of the defects. Shafeek et al. [3] developed another vision system which makes use of various image processing and computer vision algorithms applied to capture images of the radiographic films to recognize the defects and to make acceptance decisions according to international standards. This system was capable of identifying and testing the main types of welding defects in gas pipelines welded by shielded metal arc welding. They are used to capture single images for the radiographic films which are used to identify the subsurface defects only.

On the other hand, developments in image processing, computer vision, artificial intelligence, and other related fields have significantly improved the efficiency of visual inspection techniques. It was reported that about $60-90 \%$ of all existing machine vision applications were automated visual inspection. A feature is a value describing an object in a numerical form, and the selection of good features is critical to the success of any classification algorithm. Generally, 2D features are computationally simpler than 3Dfeatures [4]. Efficient techniques for solder joint inspection have been described using three layers of ring-shaped light-emitting diodes (LEDs) with different illumination angles; three frames of images were sequentially obtained, the regions segmented, and then the solder joints classified using a fuzzy membership function and neural network classifier [4]. Jagannathan [5] developed a new system for the intelligent machine vision inspection of wave solder joints. A modified intelligent histogram regarding technique was used that divides the gray level histogram of the captured images from a joint into different modes, and the neural networks was employed to identify and classify the defective solder joints. The back-propagation algorithm was employed to train the neural networks. After training, the neural network was employed to successfully identify and classify the defects of the welded joints. They used images captured with light sources of ring-shaped LEDs with different illumination angles in solder joints. In ringshaped LEDs, illuminations are focused in the center of the image. In welding images, the sizes of beads are not in circular shape and some informations about the welding beads are missed. Poor quality radiographic images have led to the development of various automatic defect detection algorithms that focus on extracting defects using various image segmentation methods [5-7]. Neural networks are used to improve the computational speed of the system for such activities as feature extraction and interpretation [6]. Two-dimensional images taken under controlled conditions of good lighting and low noise is the simplified strategy of industrial vision applications [8]. NDT is particularly important for critical applications where weld failure can be catastrophic, such as in pressure vessels, load-bearing structural members, and power plants [9]. Lashkia [10] proposed fuzzy reasoning to detect low-contrast defects using local image characteristics such as special contrast, special variance, and distance between two contrast regions. As the measurement system is optical, only the surface of the weld was mapped. A digitized radiographic image is often corrupted by non-uniform illumination, noise, and poor contrast [10]. The applied inspection criteria specified in the standards include the measurement of the height and crosssectional area of the weld together with detection of porosity density and undercuts [7]. Inspection of welds is important not only to ensure the integrity of the welded engineering artifacts but also to improve the fabrication process [11]. Da Silva et al. [12] also concluded that the lack of a high number of samples to increase the reliability of the classification is a common problem in the automatic interpretation of weld radiographs. Radiographic films usually have noise and deficient contrast due to intrinsic factors involved in the inspection technique, such as non-uniform illumination and the limited range of intensities of the image capture device [13]. Liao and $\mathrm{Li}$ [14] developed welding flaw detection based on the fitted line profiles of a weld image and successfully detected $93.33 \%$ of various defects from linear welds. Wang and Liao [15] used a set of parameters to classify six possible defects and obtained the highest accuracy of $92 \%$. In this work, 108 data sets were used for training while 12 data sets were used for testing. However, the 12 test samples used for classifying six types of defects are considered small and the success rate for individual defect was not reported. Different types of methods that characterize a shape can be viewed from a different context, such as description-based boundaries and region, local and global shape characteristics, statistical or synthetic object description, object reconstruction ability, or incomplete shape recognition ability [16].

The information capture from different viewpoints can reinforce the diagnosis when a single image is insufficient [17]. In tune with the trend, four zones of LEDs with different illumination angles are used to capture the weld joints. In this newly introduced vision system, 2D feature average gray values are extracted from the MIG welding joints and are classified using the back-propagation neural 
network as good weld, excess weld, insufficient weld, and no weld. In general, the calibration process is difficult to carry out in industrial environment due to vibrations and random movements that vary with time [17]. Therefore, any calibration process is not followed in this method. This paper is organized as follows: Section 2 represents the overview of the system. The experimentation functions are discussed in Section 3. Preprocessing of digitized image and feature extraction are discussed in Sections 4 and 5. Neural classifier is discussed in Section 6. The test results are presented in Section 7, followed by the conclusions.

\section{System overview}

The photograph of the overall inspection system is shown in Fig. 1, A RAPID 1 V3.4 machine vision system is used to capture the images. Vision-based inspection systems are a set of new technologies for non-contact inspections and measurements. The instruments integrate a multitude of technologies including digital imaging, electronics, embedded systems, and software. The Rapid-1 V3.4, a vision-based metrology instrument, utilizes these cutting-edge technologies that enable doing precise inspections. Further innovative design and creative developments have led to a wide range of hardware and software capabilities that will enhance our ability not only to inspect manufactured parts but also in our design and development. Rapid-1 is capable of carrying out diverse measurement tasks including all basic 2D measurements, depth, and even threads parameters. Its primary advantage lies in its high-resolution optics combined with precision work stage and power software.

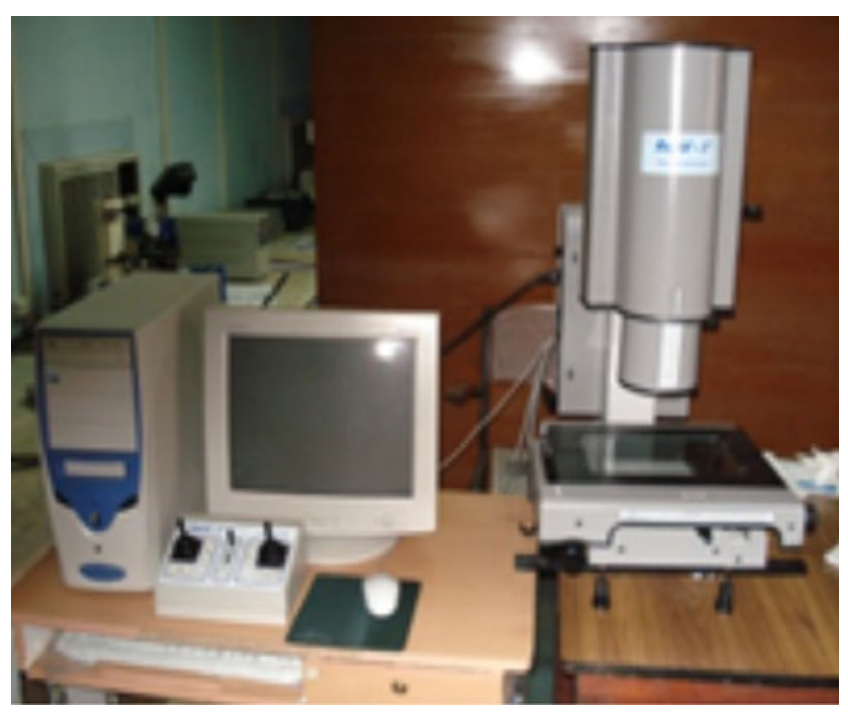

Fig. 1 RAPID 1 machine vision system
The quality of imaging cannot be changed if the hardware is not suitably designed [18]. Four zones of LEDs with different illumination angles and input camera are controlled by the host computer. Figure 2 shows the four zones of LEDs with different illumination angle positions. Full operations including image capturing and inspection software are executed in the host computer. Four frames of images are sequentially captured as four zones of LEDs are turned on, one after the other, as shown in the following figures.

\section{Experimentation}

The three main functions are carried out in this experimentation. First of all, different types of joints, like acceptable and unacceptable joints in the single $\mathrm{V}$ groove butt welding joint in the MIG welding process, have been prepared as per Standard EN 25817. A carbon steel plate (size, $80 \times 20 \times$ $4 \mathrm{~mm}$ ) is used as a parent material in this work. The voltage and current maintained during welding are $27 \mathrm{~V}$ and $260 \AA$. ER 70S6 with a 1.2-mm diameter electrode is used in this experiment. Carbon dioxide is supplied during the welding process, and standoff distance is maintained at $15 \mathrm{~mm}$.

The second one is based on the values obtained for the various measurements; acceptance or non-acceptance of the weld will be decided in conformity with the EN 25817 acceptance levels for intermediate service conditions. Figure 3 depicts the different types of acceptable and unacceptable groove weld profiles in butt joint. Figure $3 \mathrm{a}$ shows the image of good weld, where $h, b$, and $t$ denote the reinforcement height, width of the weld, and thickness of the workpiece, respectively. Good weld occurs when the reinforcement height $(h)$ is $h \leq 1 \mathrm{~mm}+0.15 b$, maximum of $7 \mathrm{~mm}$, and also the under groove height is $h_{1} \leq 0.1 t$, maximum of $1 \mathrm{~mm}$. Figure $3 \mathrm{~b}$ shows excessive reinforcement. When the reinforcement height $(h)$ of the weld lies between 1 and $7 \mathrm{~mm}$, then it is called as excessive reinforcement. Figure $3 \mathrm{c}$ shows insufficient weld. When the under groove height $\left(h_{1}\right)$ lies between 0.1 and $1 \mathrm{~mm}$, then it is called

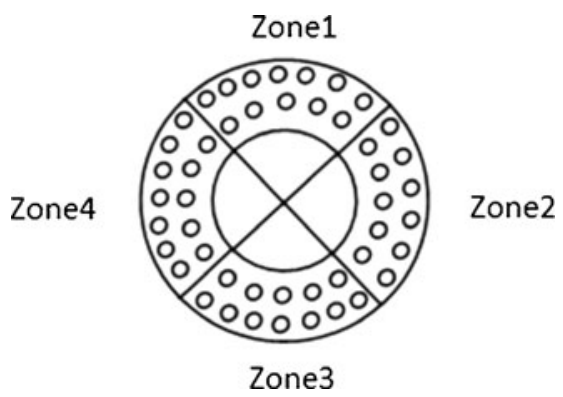

Fig. 2 Four zones of LED illumination 
Acceptable groove weld profile in butt joint

a

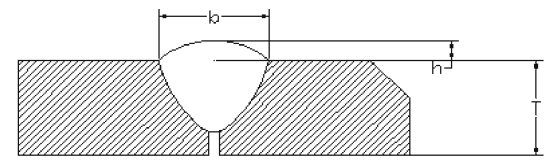

Good weld

Where, $\mathrm{h}=$ reinforcement in $\mathrm{mm}$

$\mathrm{b}=$ width of the weld in $\mathrm{mm}$

$\mathrm{t}=$ work piece thickness

Unacceptable groove weld profile in butt joint

b
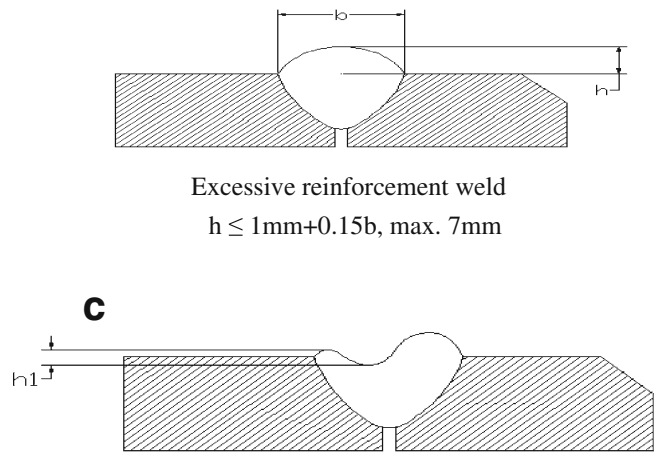

Insufficient weld

$\mathrm{h} 1 \leq 0.1 \mathrm{t}, \max .1 \mathrm{~mm}$

Where, $\mathrm{h} 1$ = under groove height

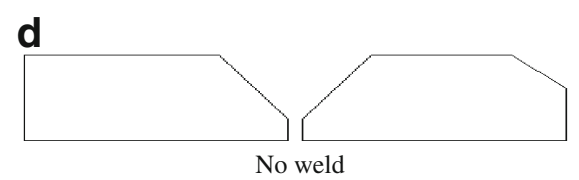

Fig. 3 Different types of acceptable and unacceptable groove weld profiles in butt joint as per EN 25817: Good weld (a), excessive reinforcement weld (b), insufficient weld (c), and no weld (d)

insufficient weld. Figure $3 d$ illustrates no weld. When the groove surface is not filled, then it is called no weld.

The types of welding defects to be inspected include defect-free welded joint (good weld), excess weld, insufficient weld, and no weld. Figure 4 shows the images of defect-free welded joint in four zones. Figure $4 a-d$ shows the top left quadrant (zone 1), the top right quadrant (zone 2), the bottom left quadrant (zone 3 ), and the bottom right quadrant (zone 4) of the image of the defect-free welded joint, respectively. Figure 5 shows the images of excess weld in four zones. In Fig. 5a-d, shown are zones 1, 2, 3, and 4 of the four quadrants of images of excess weld, respectively. Figure 6 shows images of insufficient weld in four zones. Information about zones 1, 2, 3, and 4 of the four quadrants of images of insufficient welds are shown in Fig. 6a-d, respectively. Figure 7 shows the images of no weld in four zones.
Figure $7 \mathrm{a}-\mathrm{d}$ also illustrates information on zones $1,2,3$, and 4 of the four quadrants of images of no weld. Four zones of LEDs with different illuminations are used to capture four types of weld joints and processed using the RAPID 1 V3.4 machine vision system.

\section{Preprocessing}

Four frames of images sequentially captured as four zones of LEDs are turned on, one after the other. Image processing seeks to modify and prepare the pixel values of a digitized image to produce more suitable forms for subsequent operations. In this stage, the weld region must be isolated from the rest of the parent metals. Noise on images usually appears as randomly dispersed pixels having different values of intensity in relation to their nearest pixels. Low-pass filters are usually employed to remove the noise and extend the technique of fuzzy k-means clustering followed by the cropping mechanism. The ROI has been identified (Fig. 8).

\section{Feature extraction}

A feature is a value describing an object in a numerical form; the selection of good features is critical to the success of any classification algorithm. Rather than directly using the raw data, some measures or descriptors are often selected, upon which the classes of the observed objects are determined by a classifier. These measures, commonly called features, form the feature space that is generally of a much lower dimension than the data space. The process of searching for internal structure in data items, that is, for features or properties of the data, is called feature extraction. The process of choosing desirable features from the initial set of candidates is called feature selection. The relevance of extracted features is determined either by trial and error or based on an automatic feature selection procedure [19].

The extraction of desirable features is an extremely difficult task and is very much problem-dependent [9]. In order to distinguish welds from non-welds, features with discriminating capability must be identified [20]. In this process, 2D features are the average gray levels, and the percentages of highlights of $I_{1}, I_{2}, I_{3}$, and $I_{4}$ are extracted from the digitized images of samples. The bitmap images are read and stored into an array variable. Then, true color images are converted into grayscale images. After this selection, a region of interest is cropped for further processing. Finally, average values of pixels in the cropped images are computed as follows [4]:

$x=\left(x_{1}, x_{2}, x_{3}, x_{4}\right)$ 
Fig. 4 Four images of good weld: zone 1 (a), zone 2 (b), zone 3 (c), and zone 4 (d)

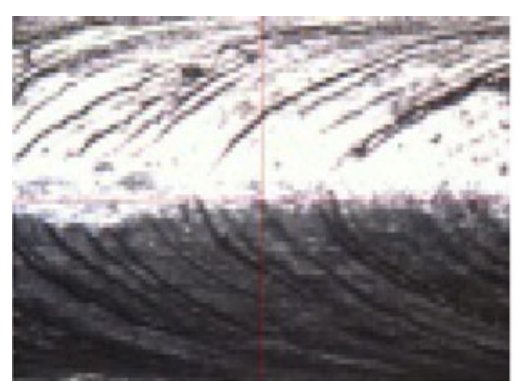

a Zone 1

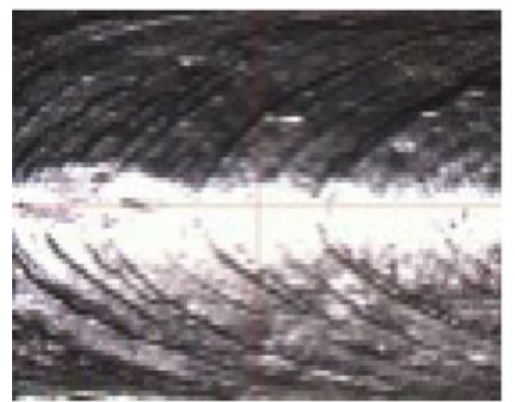

C Zone 3

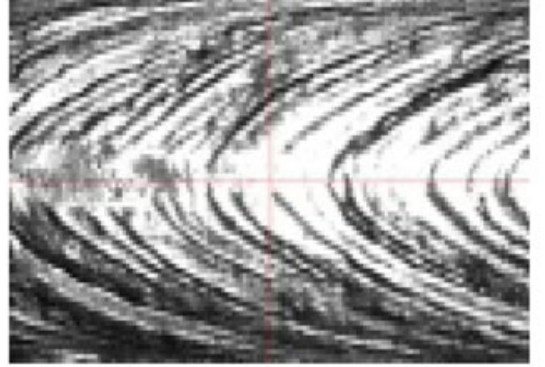

b Zone 2

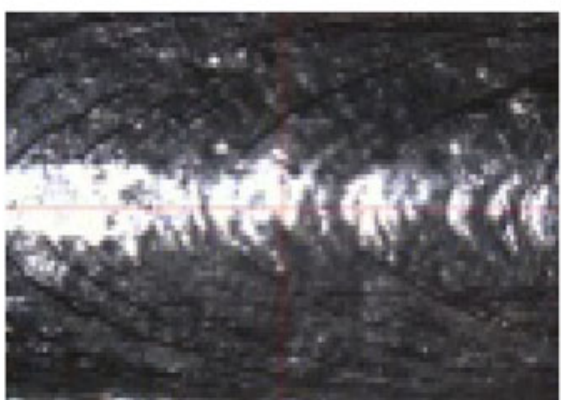

d Zone 4
$x_{1}=\frac{1}{N} \sum_{(x, y) \in R} I_{1} \quad(x, y)$

$x_{2}=\frac{1}{N} \sum_{(x, y) \in R} I_{2}(x, y)$

Fig. 5 Four images of excess weld: zone 1 (a), zone 2 (b), zone 3 (c), and zone 4 (d)

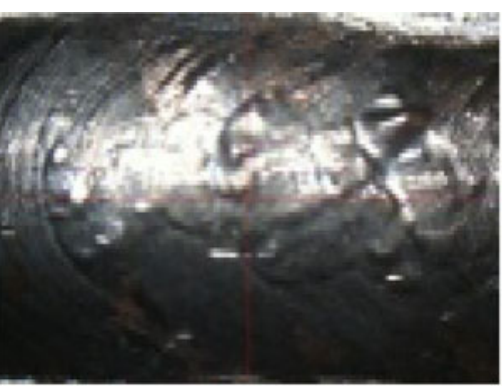

a Zone 1

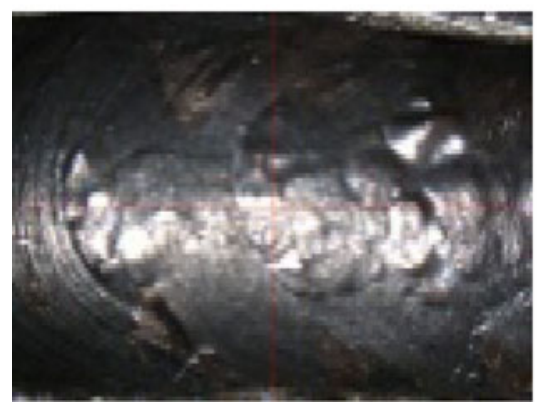

C Zone 3

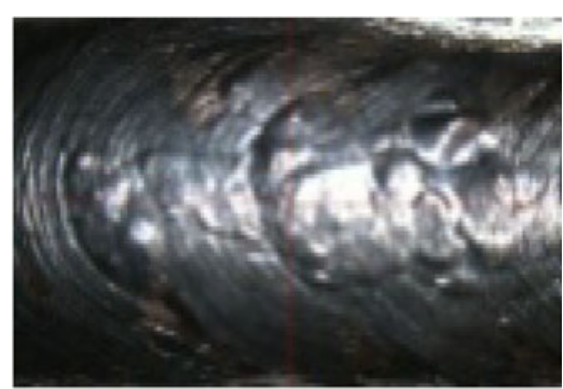

b Zone 2

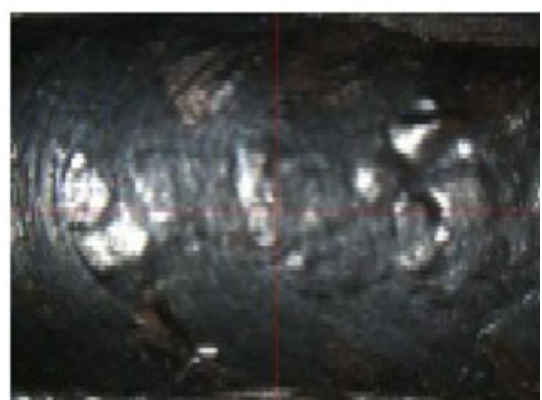

d Zone 4 
Fig. 6 Four images of

insufficient weld: zone 1 (a),

zone 2 (b), zone 3 (c), and

zone 4 (d)

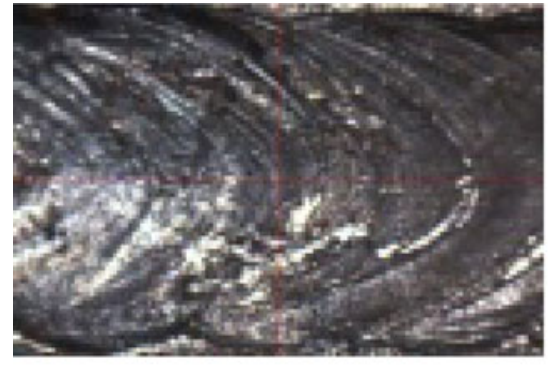

a Zone 1

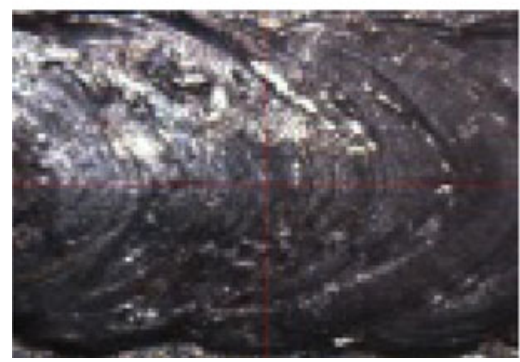

C Zone 3

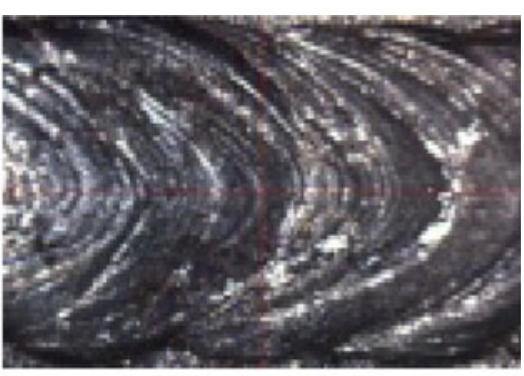

b Zone 2

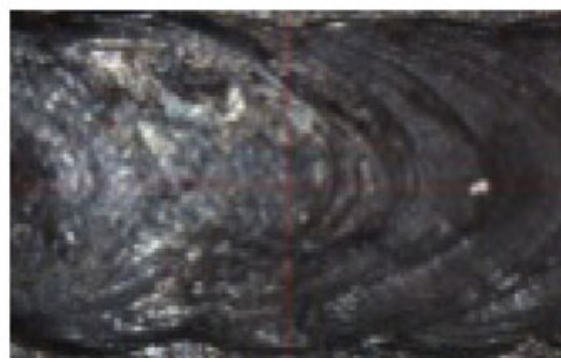

d Zone 4 where $x$ is the 2D feature vector, $x_{1}$ is the average grayscale value of zone 1 cropped image, $x_{2}$ is the average grayscale value of zone 2 cropped image, $x_{3}$ is the average grayscale value of zone 3 cropped image, $x_{4}$ is the average grayscale value of zone 4 cropped image, $I_{i}(x, y)$ is the image of the $i$ th layer, $R$ is the welded region, and $N$ is the number of pixels in the welded region.

The average gray values of pixels in the cropped images are calculated and tabulated. In this work, 80 welded image samples are taken into account for the classification process. Table 1 shows the average gray values of 80 samples.

\section{BPN classifier}

An artificial neural network (ANN) is an information processing paradigm that is inspired by the biological nervous
Fig. 7 Four images of no weld: zone 1 (a), zone 2 (b), zone 3 (c), and zone 4 (d)

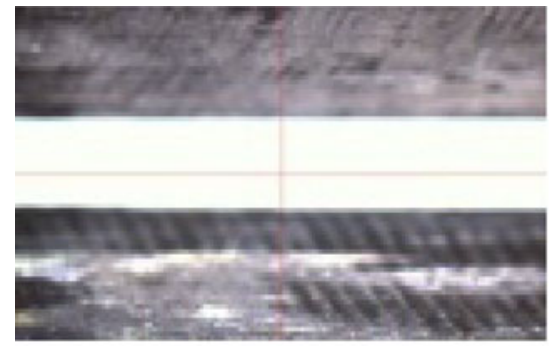

a Zone 1

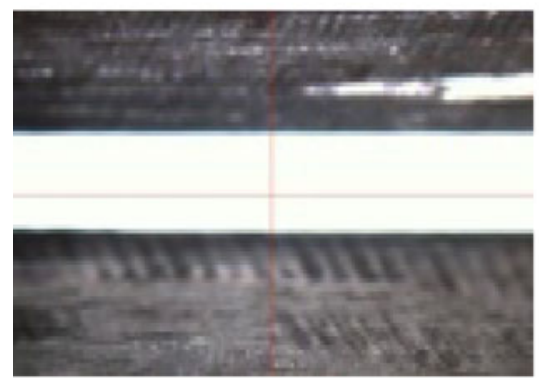

C Zone 3

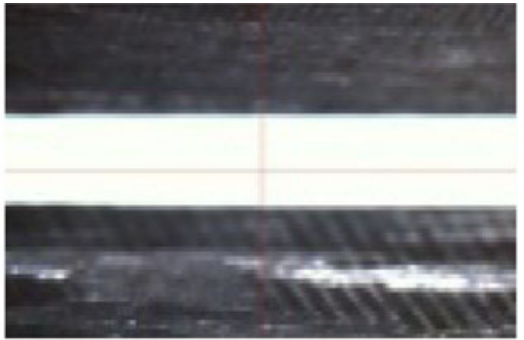

b Zone 2
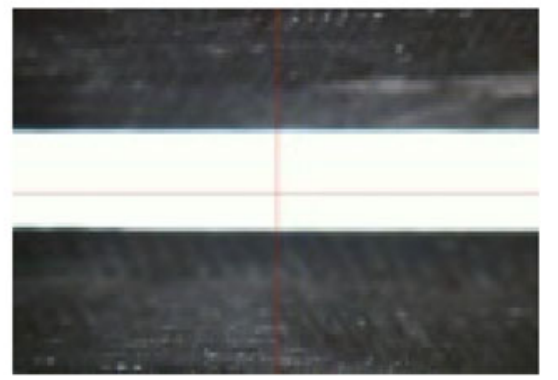

d Zone 4 


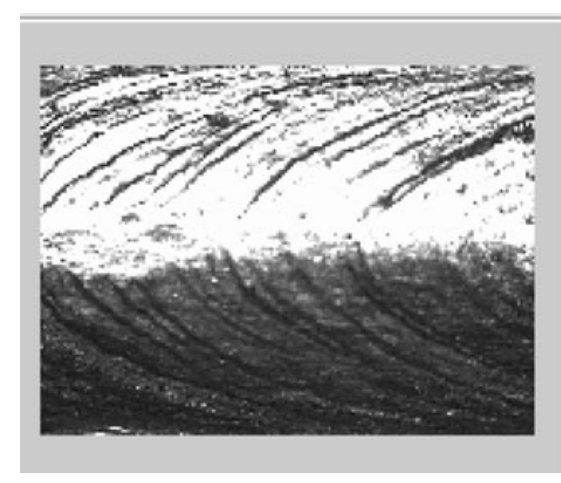

Fig. 8 Grayscale image

systems such as the brain process information. ANN has been successfully employed in similar applications to perform the classification. After feature selection, a back-propagation neural network (BPN) is employed to perform the classification [21]. A block diagram of the back-propagation neural net work is shown in Fig. 9. The back-propagation algorithm minimizes the squares of the differences between the actual output and the desired output unit for all training pairs. The error obtained when a training pair consisting of both input and output is given to the input layer of the network is given by the equation

$E_{p}=\frac{1}{2} \sum_{i}\left(T_{\mathrm{p} i}-O_{\mathrm{p} i}\right)_{2}$

where $T_{\mathrm{p} i}$ is the $i$ th component of the desired output and $O_{\mathrm{p} i}$ is the calculated output of the $i$ th neuron in the output layer.

The overall error of all the patterns is given by,

$E=\sum E_{\mathrm{p}}$

To obtain a gradient descent in $E$, the weight $W$ has to be updated

$W_{i j}=\eta \partial_{p j} O_{p i}$

where $\eta$ is a constant real number called learning rate which determines the influence of error over weight change, $\partial_{p j}$ is the error due to the $p$ th pattern connected to the $j$ th neuron, and $O_{p i}$ is the $i$ th neuron output when $p$ th is processed by the network.

In the gradient descent, Eq. 2.3, the error value $p_{j}$ can be computed as follows:

$\partial_{p j}=O_{p i}\left(1-O_{p i}\right)\left(T_{p j}-O_{p i}\right)$

For hidden layers,

$\partial_{p i}=O_{p i}\left(1-O_{p i}\right) \sum \partial_{p j} W_{j k}$
Table 1 Average gray values

\begin{tabular}{|c|c|c|c|c|}
\hline Sample no. & Zone 1 & Zone 2 & Zone 3 & Zone 4 \\
\hline 1 & 168.94258 & 124.0809 & 144.12504 & 120.6365 \\
\hline 2 & 169.09755 & 125.4698 & 141.8849 & 119.33363 \\
\hline 3 & 169.06311 & 128.4559 & 143.09225 & 119.28537 \\
\hline 4 & 163.98381 & 131.1469 & 143.95048 & 117.99456 \\
\hline 5 & 163.62224 & 130.5913 & 145.11419 & 118.7787 \\
\hline 6 & 163.01961 & 131.0774 & 144.13958 & 119.61109 \\
\hline 7 & 166.01553 & 137.015 & 144.80872 & 116.7882 \\
\hline 8 & 157.71648 & 135.4525 & 138.35014 & 113.33799 \\
\hline 9 & 161.65939 & 138.9074 & 143.87775 & 114.08594 \\
\hline 10 & 152.72327 & 133.317 & 138.48106 & 110.82875 \\
\hline 11 & 129.23798 & 102.1362 & 106.18821 & 95.954272 \\
\hline 12 & 137.65756 & 106.2682 & 113.85412 & 99.923213 \\
\hline 13 & 158.62903 & 114.4454 & 129.91327 & 109.93604 \\
\hline 14 & 147.98833 & 110.1571 & 122.14553 & 104.45915 \\
\hline 15 & 161.62495 & 116.4419 & 131.90612 & 113.21736 \\
\hline 16 & 159.54158 & 115.2787 & 132.35706 & 113.1691 \\
\hline 17 & 162.38254 & 117.4315 & 134.33536 & 114.77357 \\
\hline 18 & 171.78355 & 120.7475 & 141.78308 & 118.06694 \\
\hline 19 & 172.17956 & 122.1538 & 145.4633 & 120.2384 \\
\hline 20 & 96.145066 & 90.2785 & 86.026996 & 68.992014 \\
\hline 21 & 91.169077 & 107.987 & 89.532661 & 62.031288 \\
\hline 22 & 90.928026 & 102.1119 & 81.110336 & 62.079543 \\
\hline 23 & 82.542881 & 92.65699 & 76.135491 & 57.242019 \\
\hline 24 & 82.077996 & 92.27504 & 68.222288 & 56.228673 \\
\hline 25 & 80.924393 & 86.63264 & 64.8039 & 55.927081 \\
\hline 26 & 77.859597 & 94.80979 & 63.989306 & 53.84007 \\
\hline 27 & 80.61447 & 104.862 & 61.065493 & 52.006395 \\
\hline 28 & 76.120583 & 92.13615 & 59.479943 & 51.487658 \\
\hline 29 & 78.841021 & 83.54234 & 60.061797 & 51.415276 \\
\hline 30 & 101.36211 & 114.9836 & 84.208704 & 57.543611 \\
\hline 31 & 100.65617 & 98.6119 & 82.710432 & 67.640886 \\
\hline 32 & 100.65617 & 98.6119 & 82.710432 & 67.640886 \\
\hline 33 & 109.90221 & 92.72644 & 85.69243 & 84.264595 \\
\hline 34 & 107.23343 & 81.58051 & 87.830741 & 92.70915 \\
\hline 35 & 105.52885 & 79.4798 & 104.07899 & 101.47942 \\
\hline 36 & 100.05354 & 70.33042 & 99.045961 & 102.92706 \\
\hline 37 & 99.089337 & 73.97629 & 88.121667 & 89.729429 \\
\hline 38 & 99.244298 & 72.58739 & 88.470779 & 80.404227 \\
\hline 39 & 93.855078 & 77.83048 & 86.652488 & 79.342626 \\
\hline 40 & 94.474925 & 73.94156 & 82.099487 & 76.628305 \\
\hline 41 & 89.619461 & 79.63605 & 86.987053 & 72.864446 \\
\hline 42 & 89.12014 & 92.91741 & 89.547207 & 60.873178 \\
\hline 43 & 91.909449 & 95.88618 & 83.423203 & 57.14551 \\
\hline 44 & 97.21258 & 100.0876 & 80.470298 & 55.746127 \\
\hline 45 & 93.321322 & 89.94864 & 86.536117 & 64.323382 \\
\hline 46 & 84.075279 & 98.03898 & 88.965354 & 62.224307 \\
\hline 47 & 83.954753 & 95.55632 & 91.496416 & 64.817991 \\
\hline 48 & 80.373419 & 88.94168 & 84.645094 & 61.367788 \\
\hline 49 & 77.859597 & 89.91391 & 75.379082 & 52.766405 \\
\hline
\end{tabular}


Table 1 (continued)

\begin{tabular}{|c|c|c|c|c|}
\hline Sample no. & Zone 1 & Zone 2 & Zone 3 & Zone 4 \\
\hline 50 & 71.833312 & 93.73339 & 62.34557 & 43.043103 \\
\hline 51 & 130.71872 & 152.6748 & 109.81025 & 70.138061 \\
\hline 52 & 58.79932 & 42.95173 & 52.090408 & 50.607012 \\
\hline 53 & 69.285055 & 48.90664 & 61.472791 & 59.739195 \\
\hline 54 & 74.20939 & 45.15661 & 64.993002 & 74.480975 \\
\hline 55 & 86.451357 & 56.00739 & 72.600733 & 75.108285 \\
\hline 56 & 94.388835 & 64.18454 & 74.59358 & 76.797196 \\
\hline 57 & 96.007323 & 68.16027 & 78.113792 & 70.668862 \\
\hline 58 & 106.30366 & 78.88952 & 79.830259 & 67.544376 \\
\hline 59 & 110.12605 & 87.32709 & 85.837893 & 71.658081 \\
\hline 60 & 107.38839 & 93.95909 & 83.248647 & 71.851099 \\
\hline 61 & 142.90903 & 154.1679 & 122.24736 & 94.59108 \\
\hline 62 & 143.61497 & 158.1263 & 124.21111 & 84.84365 \\
\hline 63 & 147.00691 & 173.6125 & 127.07674 & 73.383183 \\
\hline 64 & 142.35806 & 171.8243 & 117.15614 & 75.675276 \\
\hline 65 & 139.44823 & 155.6436 & 121.91279 & 88.076709 \\
\hline 66 & 135.50531 & 166.3902 & 115.52695 & 72.406027 \\
\hline 67 & 131.45909 & 168.1437 & 114.49416 & 64.528464 \\
\hline 68 & 132.78488 & 160.7652 & 121.92734 & 77.002278 \\
\hline 69 & 129.15189 & 156.4943 & 116.9234 & 68.412959 \\
\hline 70 & 129.68564 & 150.2616 & 116.5161 & 72.466346 \\
\hline 71 & 70.059863 & 91.37226 & 64.658437 & 44.599314 \\
\hline 72 & 129.28963 & 116.5287 & 108.96656 & 100.7556 \\
\hline 73 & 143.59775 & 130.1747 & 117.21433 & 105.32773 \\
\hline 74 & 147.81615 & 138.4039 & 117.22887 & 101.09339 \\
\hline 75 & 146.28375 & 142.831 & 121.25821 & 101.08132 \\
\hline 76 & 145.25068 & 151.0082 & 123.70199 & 98.946057 \\
\hline 77 & 145.44007 & 151.4769 & 126.96037 & 90.175784 \\
\hline 78 & 144.33813 & 151.9804 & 123.58562 & 89.512283 \\
\hline 79 & 143.28783 & 153.5082 & 121.33094 & 86.641134 \\
\hline 80 & 147.62675 & 152.6054 & 120.95273 & 89.608792 \\
\hline
\end{tabular}

In this work, a BPN classifier is used to classify the weld joints. The back-propagation algorithm was used to train the

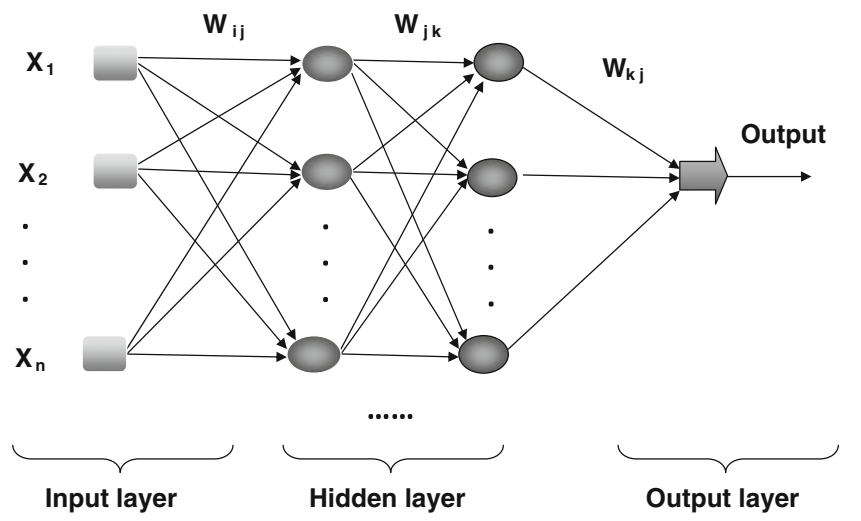

Fig. 9 Block diagram of back-propagation neural net work network. The network was trained by using the average gray values for four zones of images as input variables and the types of weld joint as output variables.

In order to improve the performance of the system, normalizing the data is important. It can make the neural network training more efficient due to a significant reduction of the dimensionality of the input data. Normalization is done as follows.

$x_{1}=\frac{x_{1}}{x_{\max }}$

where $x_{1}$ is the average grayscale value of zone 1 image and $x_{\max }$ is the maximum grayscale value of all zone images.

\subsection{Input variables}

The average gray values of four zones of images for four types of welded joints are used as input variables as follows.

\begin{tabular}{llll}
\hline Good weld & Excess weld & Insufficient & No weld \\
Zone 1 & Zone 1 & Zone 1 & Zone 1 \\
Zone 2 & Zone 2 & Zone 2 & Zone 2 \\
Zone 3 & Zone 3 & Zone 3 & Zone 3 \\
Zone 4 & Zone 4 & Zone 4 & Zone 4
\end{tabular}

\subsection{Output variables}

Four types of joints - good weld, excess weld, insufficient, and no weld - are used as output variables. Table 2 shows the inputs and outputs of the training samples.

- Features like the average gray values of four zones for four types of weld joints are the inputs given to the input layer of ANN.

- The weights between the input layer and the hidden layer and the weights between the hidden layer and the output layer are generated randomly for the selected topology 4-5-5-1 of the network.

- $\quad$ The number of training patterns used for training is 80 .

- The patterns were normalized.

- The training was done off-line using the computer.

The training function TRAINLM is used in this network. The application of Leven berg-Marquardt to neural network training is the fastest method for training a moderate-sized feed-forward neural network. In many cases, TRAINLM is able to obtain lower mean square errors than any of the other algorithms tested. The number of iterations in this work is 5,000 , the learning rate is 0.0001 , the hyperbolic tangent function is an activation function, and a three-layer feedforward BPN is used. 
Table 2 Inputs and outputs of the training samples

\begin{tabular}{lllllll}
\hline Type of & Sample & Zone 1 & Zone 2 & Zone 3 & Zone 4 & Output
\end{tabular}
welding no.

\begin{tabular}{|c|c|c|c|c|c|c|}
\hline \multirow[t]{20}{*}{ No weld } & 1 & 0.9812 & 0.7147 & 0.9908 & 1 & 1 \\
\hline & 2 & 0.9821 & 0.7227 & 0.9754 & 0.9892 & 1 \\
\hline & 3 & 0.9819 & 0.7399 & 0.9837 & 0.9888 & 1 \\
\hline & 4 & 0.9524 & 0.7554 & 0.9896 & 0.9781 & 1 \\
\hline & 5 & 0.9503 & 0.7522 & 0.9976 & 0.9846 & 1 \\
\hline & 6 & 0.9468 & 0.755 & 0.9909 & 0.9915 & 1 \\
\hline & 7 & 0.9642 & 0.7892 & 0.9955 & 0.9681 & 1 \\
\hline & 8 & 0.916 & 0.7802 & 0.9511 & 0.9395 & 1 \\
\hline & 9 & 0.9389 & 0.8001 & 0.9891 & 0.9457 & 1 \\
\hline & 10 & 0.887 & 0.7679 & 0.952 & 0.9187 & 1 \\
\hline & 11 & 0.7506 & 0.5883 & 0.73 & 0.7954 & 1 \\
\hline & 12 & 0.7995 & 0.6121 & 0.7827 & 0.8283 & 1 \\
\hline & 13 & 0.9213 & 0.6592 & 0.8931 & 0.9113 & 1 \\
\hline & 14 & 0.8595 & 0.6345 & 0.8397 & 0.8659 & 1 \\
\hline & 15 & 0.9387 & 0.6707 & 0.9068 & 0.9385 & 1 \\
\hline & 16 & 0.9266 & 0.664 & 0.9099 & 0.9381 & 1 \\
\hline & 17 & 0.9431 & 0.6764 & 0.9235 & 0.9514 & 1 \\
\hline & 18 & 0.9977 & 0.6955 & 0.9747 & 0.9787 & 1 \\
\hline & 19 & 1 & 0.7036 & 1 & 0.9967 & 1 \\
\hline & 20 & 0.5584 & 0.52 & 0.5914 & 0.5719 & 0.75 \\
\hline \multirow{20}{*}{$\begin{array}{l}\text { Insufficient } \\
\text { weld }\end{array}$} & 21 & 0.5295 & 0.622 & 0.6155 & 0.5142 & 0.75 \\
\hline & 22 & 0.5281 & 0.58816 & 0.5576 & 0.5146 & 0.75 \\
\hline & 23 & 0.4794 & 0.5337 & 0.5234 & 0.4745 & 0.75 \\
\hline & 24 & 0.4767 & 0.5315 & 0.469 & 0.4661 & 0.75 \\
\hline & 25 & 0.47 & 0.499 & 0.4455 & 0.4636 & 0.75 \\
\hline & 26 & 0.4522 & 0.5461 & 0.4399 & 0.4463 & 0.75 \\
\hline & 27 & 0.4682 & 0.604 & 0.4198 & 0.4311 & 0.75 \\
\hline & 28 & 0.4421 & 0.5307 & 0.4089 & 0.4268 & 0.75 \\
\hline & 29 & 0.4579 & 0.4812 & 0.4129 & 0.4262 & 0.75 \\
\hline & 30 & 0.5887 & 0.6623 & 0.5789 & 0.477 & 0.75 \\
\hline & 31 & 0.5846 & 0.568 & 0.5686 & 0.5607 & 0.75 \\
\hline & 32 & 0.5846 & 0.568 & 0.5686 & 0.5607 & 0.75 \\
\hline & 33 & 0.6383 & 0.5341 & 0.5891 & 0.6985 & 0.75 \\
\hline & 34 & 0.6228 & 0.4699 & 0.6038 & 0.7685 & 0.75 \\
\hline & 35 & 0.6129 & 0.4578 & 0.7155 & 0.8412 & 0.75 \\
\hline & 36 & 0.5811 & 0.4051 & 0.6809 & 0.8532 & 0.75 \\
\hline & 37 & 0.5755 & 0.4261 & 0.6058 & 0.7438 & 0.75 \\
\hline & 38 & 0.5764 & 0.4181 & 0.6082 & 0.6665 & 0.75 \\
\hline & 39 & 0.5451 & 0.4483 & 0.5957 & 0.6577 & 0.75 \\
\hline & 40 & 0.5487 & 0.4259 & 0.5644 & 0.6352 & 0.75 \\
\hline \multirow[t]{8}{*}{ Excess weld } & 41 & 0.5176 & 0.5352 & 0.6156 & 0.5046 & 0.5 \\
\hline & 42 & 0.5338 & 0.5523 & 0.5735 & 0.4737 & 0.5 \\
\hline & 43 & 0.5646 & 0.5765 & 0.5532 & 0.4621 & 0.5 \\
\hline & 44 & 0.542 & 0.5181 & 0.5949 & 0.5332 & 0.5 \\
\hline & 45 & 0.4883 & 0.5647 & 0.6116 & 0.5158 & 0.5 \\
\hline & 46 & 0.4876 & 0.5504 & 0.629 & 0.5373 & 0.5 \\
\hline & 47 & 0.4668 & 0.5123 & 0.5819 & 0.5087 & 0.5 \\
\hline & 48 & 0.4522 & 0.5179 & 0.5182 & 0.4374 & 0.5 \\
\hline
\end{tabular}

Table 2 (continued)

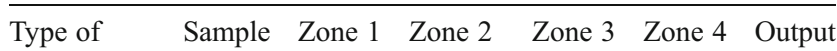
welding no.

\begin{tabular}{|c|c|c|c|c|c|c|}
\hline & 49 & 0.4172 & 0.5399 & 0.4286 & 0.3568 & 0.5 \\
\hline & 50 & 0.4069 & 0.5263 & 0.4445 & 0.3697 & 0.5 \\
\hline & 51 & 0.3415 & 0.2474 & 0.3581 & 0.4195 & 0.5 \\
\hline & 52 & 0.4024 & 0.2817 & 0.4226 & 0.4952 & 0.5 \\
\hline & 53 & 0.431 & 0.2601 & 0.4468 & 0.6174 & 0.5 \\
\hline & 54 & 0.5021 & 0.3226 & 0.4991 & 0.6226 & 0.5 \\
\hline & 55 & 0.5482 & 0.3697 & 0.5128 & 0.6366 & 0.5 \\
\hline & 56 & 0.5576 & 0.3926 & 0.537 & 0.5858 & 0.5 \\
\hline & 57 & 0.6174 & 0.4544 & 0.5488 & 0.5599 & 0.5 \\
\hline & 58 & 0.6396 & 0.503 & 0.5901 & 0.594 & 0.5 \\
\hline & 59 & 0.6237 & 0.5412 & 0.5723 & 0.5956 & 0.5 \\
\hline & 60 & 0.5205 & 0.4587 & 0.598 & 0.604 & 0.5 \\
\hline Good weld & 61 & 0.8341 & 0.9108 & 0.8539 & 0.7033 & 0.25 \\
\hline & 62 & 0.8538 & 1 & 0.8736 & 0.6083 & 0.25 \\
\hline & 63 & 0.8268 & 0.9897 & 0.8054 & 0.6273 & 0.25 \\
\hline & 64 & 0.8099 & 0.8965 & 0.8381 & 0.7301 & 0.25 \\
\hline & 65 & 0.787 & 0.9584 & 0.7942 & 0.6002 & 0.25 \\
\hline & 66 & 0.7635 & 0.9685 & 0.7871 & 0.5349 & 0.25 \\
\hline & 67 & 0.7712 & 0.926 & 0.8382 & 0.6383 & 0.25 \\
\hline & 68 & 0.7501 & 0.9014 & 0.8038 & 0.5671 & 0.25 \\
\hline & 69 & 0.7532 & 0.8655 & 0.801 & 0.6007 & 0.25 \\
\hline & 70 & 0.7592 & 0.8794 & 0.7549 & 0.5814 & 0.25 \\
\hline & 71 & 0.7509 & 0.6712 & 0.7491 & 0.8352 & 0.25 \\
\hline & 72 & 0.834 & 0.7498 & 0.8058 & 0.8731 & 0.25 \\
\hline & 73 & 0.8585 & 0.7972 & 0.8059 & 0.838 & 0.25 \\
\hline & 74 & 0.8496 & 0.8227 & 0.8336 & 0.8379 & 0.25 \\
\hline & 75 & 0.8436 & 0.8698 & 0.8504 & 0.8202 & 0.25 \\
\hline & 76 & 0.8447 & 0.8725 & 0.8728 & 0.7475 & 0.25 \\
\hline & 77 & 0.8383 & 0.8754 & 0.8496 & 0.742 & 0.25 \\
\hline & 78 & 0.8322 & 0.8842 & 0.8341 & 0.7182 & 0.25 \\
\hline & 79 & 0.8574 & 0.879 & 0.8315 & 0.7428 & 0.25 \\
\hline & 80 & 0.83 & 0.888 & 0.8404 & 0.7841 & 0.25 \\
\hline
\end{tabular}

\section{Results and discussion}

In this work, the 80 weld joint samples are used for training and testing, respectively. In each set, 20 images are used for each type, giving a total of 80 images for one good and three defect

Table 3 Training and test data of the four types of weld

\begin{tabular}{llcc}
\hline Sample no. & Type of weld & Training data & Test data \\
\hline 1 & Good & 20 & 20 \\
2 & Excess weld & 20 & 20 \\
3 & Insufficient weld & 20 & 20 \\
4 & No weld & 20 & 20 \\
& Total & 80 & 80 \\
\hline
\end{tabular}


Table 4 Classification performance of different types of welding images

\begin{tabular}{|c|c|c|c|c|c|c|}
\hline \multirow[t]{2}{*}{ Sample no. } & \multirow[t]{2}{*}{ Class } & \multirow[t]{2}{*}{ Number } & \multicolumn{4}{|l|}{ Result } \\
\hline & & & Correct & Incorrect & Correct $(\%)$ & Incorrect $(\%)$ \\
\hline 1 & Good weld & 20 & 19 & 1 & 95 & 5 \\
\hline 2 & Excess weld & 20 & 19 & 1 & 95 & 5 \\
\hline 3 & Insufficient weld & 20 & 20 & 0 & 100 & 0 \\
\hline \multirow[t]{2}{*}{4} & No weld & 20 & 18 & 2 & 90 & 10 \\
\hline & Total & 80 & 76 & 4 & 95 & 5 \\
\hline
\end{tabular}

types training. For testing of the sample, 80 images are used in the BPN network. The training data fed into the neural network are the average gray values of four images for four zones as input variables and the type of weld joint as the output variable. Table 3 shows the training and test data of the four types of weld: good, excess, insufficient, and no weld.

Networks with different topologies have been tried. It is found that a 4-5-5-1 architecture offers a more accurate prediction than any other network structure. The average training error depends upon the iteration number. The performance of the trained BPN that can be reiterated using a set of unseen patterns is known as testing or validation. The second group of data obtained is used for validation. The accuracy of the BPN is determined by means of recognition rate. The recognition rate is mostly dependent on the number of hidden neurons and the learning rate used in the network. The recognition rate is defined as follows:

Recognition rate $=\frac{\text { Number of unseen patterns correctly classified }}{\text { Total number of unseen patterns }} \times 100$

The network was trained at 0.0001 allowable errors; it can be seen that the error coverage was $7.8222 \mathrm{e}-005$. The performance of the proposed classifier has been evaluated in terms of the recognition rate and the execution time. The classification performance of an individual defect type is shown in Table 4. For individual comparison, it was found that the accuracy varies with the type of defect. The highest accuracy is $100 \%$ for insufficient weld and the lowest is for no weld $(90 \%)$. The overall accuracy is $95 \%$. As a matter of fact, a $2 \mathrm{D}$ feature shows a significant difference in the comparison test. The performance of a BPN network using 80 samples is shown in Fig. 10.

\section{Conclusion}

The technique used in this method for welding joint inspections using 2D feature extraction of images by a machine vision system has been developed and verified with real welding defects. Four zones of LEDs are used for the efficient extraction of shape information which is used to characterize weld defects, and it can be classified into one of the predefined ones based on the backpropagation neural network. The classification of real defects using this method provides the highest overall accuracy of $95 \%$. This same method was used in single image captured by a front light illumination system and which was performed only in a 92.5\% accuracy level. Thus, the proposed method overcomes the problem of inaccuracy in the images, non-uniformed illumination, noise and deficient contrast, and confusion in defects
Fig. 10 Classification performance of BPN network using 80 samples

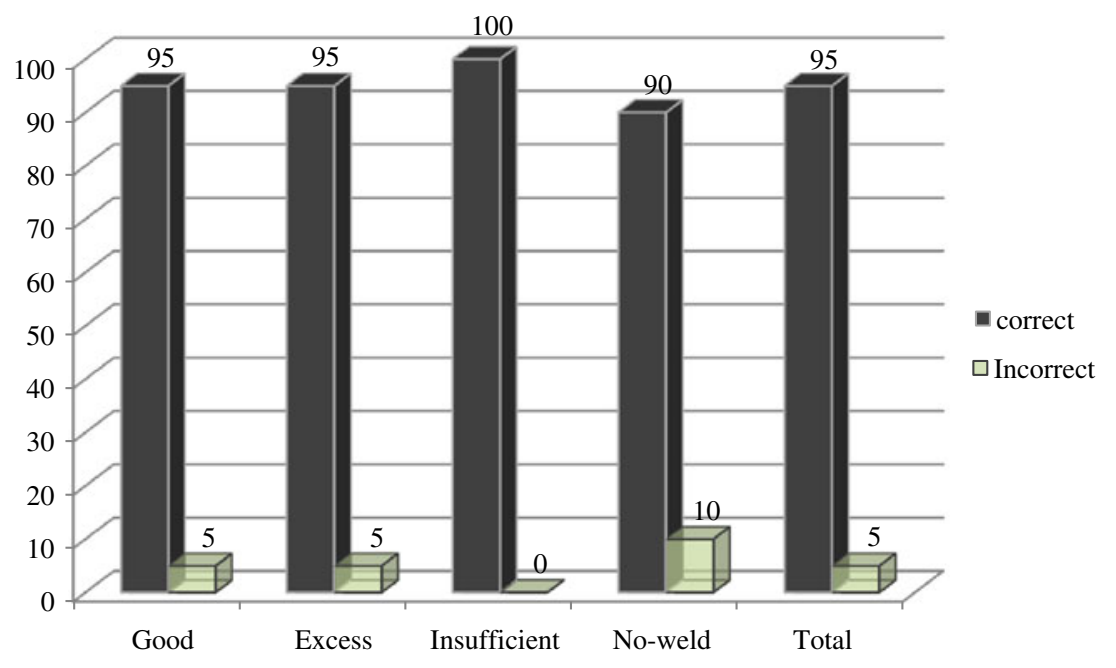


if they occur in the same spot at the surface and the subsurface in a certain level. Future work is to be extended to extract 3D features through the distribution of illuminations in different tilt angles. The estimation of tilt angle at each pixel position $(x, y)$ gives a 3D shape information of the welded joints, and the reflected slant angle measures the angle between the $x$-axis and projection of the $x y$ plane. This 3D feature extraction approach will increase the classification accuracy. It can be used in computer-aided inspection of welding defects in manufacturing systems. This vision-based inspection system could be further used for the classification of images with different joints in the welding process.

Open Access This article is distributed under the terms of the Creative Commons Attribution Noncommercial License which permits any noncommercial use, distribution, and reproduction in any medium, provided the original author(s) and source are credited.

\section{References}

1. Shafeek HI, Gadelmawla ES, Abdel-Shafy AA, Elewa IM (2004) Assessment of welding defects for gas pipeline radiographs using computer vision. NDT\&E International 37:291-299

2. Warren Liao T (2009) Improving the accuracy of computer-aided radiographic weld inspection by feature selection. NDT\&E International 42:229-239

3. Shafeek HI, Gadelmawla ES, Abdel-Shafy AA, Elewa IM (2004) Automatic inspection of gas pipeline welding defects using an expert vision system. NDT\&E International 37:301-307

4. Kim T-H, Cho T-H, Moon YS, Park SH (1999) Visual inspection system for the classification of solder joints. Pattern Recognition 32:565-575

5. Jagannathan S (1997) Automatic inspection of wave soldered joints using neural networks. J Manuf Syst 16(6):389-398

6. Jagannathan S (1992) Intelligent inspection of wave soldered jointstechnical report. Journal of Manufacturing Systems 11(2):137-143
7. Gauss M, Buerkle A, Laengle T, Woern H, Stelter J, Ruhmkorf S, Middelmann R (2003) Adaptive robot based visual inspection of complex parts. ISR2003

8. Malamas EN, Petrakis EGM, Zervakis M, Petit L (2003) A survey on industrial vision systems, applications and tools. Image and Computing 21:171-188

9. Liao TW, Li D-M, Li Y-M (1999) Detection of welding flaws from radiographic images with fuzzy clustering methods. Fuzzy Set Syst 108:145-158

10. Lashkia V (2001) Defect detection in X-ray images using fuzzy reasoning. Image and Vision Computing 19:261-269

11. Liao TW (2003) Classification of welding flaw types with fuzzy expert systems. Expert Systems with Application 25:101-111

12. da Silva RR, Caloba LP, Siqueira MHS, Rebello JMA (2004) Pattern recognition of weld defects detected by radiographic test. NDT\&E International 37:461-470

13. Vilar R, Zapata J, Ruiz R (2009) An automatic system of classification of weld defects in radiographic images. NDT\&E International 42:467-476

14. Liao TW, Li YM (1998) An automated radiographic NDT system, for weld inspection: Part II-Flaw detection. NDT\&E International 31(3):183-192

15. Wang G, Liao TW (2002) Automatic identification of different types of welding defects in radiographic images. NDT \&E International 35:519-528

16. Sonka M, Hilavac H, Boyle R (1998) Image processing, analysis and machine vision, 2nd edn. PWS Publishing, Pacific Grove

17. Carrasco M, Merry D (2011) Automatic multiple view inspection using geometrical tracking and feature analysis in aluminum wheels. Machine Vision and Applications 22:157-170

18. Wang Y, Sun Y, Lv P, Wang H (2008) Detection of line weld defects based on multiple thresholds and support vector machine. NDT\&E International 41:517-524

19. Waren Liao T, Li Damin, Li Yueming (2000) Extraction of welds from radiographic images using fuzzy classifiers. Information Sciences 126:21-40

20. Waren Liao T, Tang K (1997) Automated extraction of welds from digitized radiographic images based on MLP neural networks. Appl Artif Intell 11:197-218

21. Garcia-Allende PB, Mirapeix J, Conde OM, Cobo A, LopezHiguera JM (2009) Spectral processing technique based on feature selection and artificial neural networks for arc-welding quality monitoring. NDT\&E International 42:56-63 